\title{
El uso del suelo y el recurso escaso del agua: un caso de estudio en la periferia rural de Chile
}

\author{
EDUARDO JAIME MUÑOZ
}

Facultad de Filosofía, Humanidades y Arte, Universidad Nacional de San Juan, Argentina ejaime18@gmail.com

\section{RESUMEN}

Se presenta un estudio geográfico en el sector de Pedregal, comuna de Monte Patria, provincia del Limarí, Región de Coquimbo, Chile. El objetivo del trabajo es conocer y analizar el uso del espacio territorial en la unidad de estudio. Metodológicamente, se realizan salidas de campo, se toman fotografías de las actividades productivas que existen en el lugar, se describe el uso del territorio en cada una de las unidades del paisaje y con la ayuda del Google Earth y Arc Map (IO.I) se confeccionan mapas y se realizan entrevistas a lugareños de la zona. Los resultados del estudio darán a conocer a las comunidades los usos del espacio para orientar de mejor manera políticas de desarrollo rural en la zona.

PALABRAS CLAVE: usos del suelo; ordenamiento territorial; medio ambiente

Recepción: 05/09/2020 | Evaluación: 23/11/2020 | Aceptación: 11/12/2020 


\section{ABSTRACT}

\section{LAND USE AND SCARCE WATER RESOURCE: A CASE STUDY IN THE RURAL PERIPHERY OF CHILE}

A geographical study is presented in the Pedregal sector, Monte Patria commune, Limarí province, Coquimbo Region, Chile. The objective of the work is to know and analyze the use of territorial space in the study unit. Methodologically, field trips are carried out, photographs are taken of the productive activities that exist in the place, the use of the territory in each of the landscape units is described and with the help of Google Earth and Arc Map (IO.I) maps are made and interviews are conducted with locals in the area. The results of the study will inform the communities about the uses of the space to better guide rural development policies in the area.

KEYWORDS: land uses; territorial ordering; environment 


\section{RESUMO}

O USO DA TERRA E OS POUCOS RECURSOS DE ÁGUA: UM ESTUDO DE CASO NA PERIFERIA RURAL DO CHILE.

Um estudo geográfico é apresentado no setor Pedregal, município de Monte Patria, província de Limarí, região de Coquimbo, Chile. O objetivo do trabalho é conhecer e analisar o uso do espaço territorial na unidade de estudo. Metodologicamente, são realizadas viagens de campo, são feitas fotografias das atividades produtivas existentes no local, descreve-se o uso do território em cada uma das unidades de paisagem e com o auxílio do Google Earth e do Arc Map (IO.I) são feitos mapas e as entrevistas são realizadas com os moradores da área. Os resultados do estudo irão informar as comunidades sobre os usos do espaço para melhor orientar as políticas de desenvolvimento rural na área.

PALAVRAS-CHAVE: usos da terra; ordenamento territorial; meio ambiente 


\section{Introducción}

Los cambios en el uso del territorio generan consecuencias directas e indirectas sobre el sistema social, económico, cultural y político (Paruelo et al., 20I4). Considerando este aspecto, el sector de Pedregal, pueblo rural ubicado en el valle de Mostazal, es una zona que en el último tiempo ha ido transformando su espacio con la llegada de actividades productivas y de servicios cambiando el uso del territorio.

Por esta razón se ha planteado esta pregunta de investigación ¿Cuáles son las actividades que han ido cambiando el uso del espacio en la localidad de Pedregal? En la actualidad, el pueblo de Pedregal cuenta con 364 personas (CENSO, 2017) y las actividades productivas que allí se practican son la ganadería caprina, la agricultura, la apicultura y las actividades de servicios como la telefonía celular y un turismo incipiente, estas actividades se han venido instalando en la unidad territorial, cambiando aspectos del paisaje rural.

Según el Informe de política nacional de desarrollo rural 20I4-2024 (Gobierno de Chile, 2018), "las bajas densidades y la gran dispersión entre los distintos asentamientos, plantean una serie de importantes desafíos para asegurar el bienestar social de la población rural". En este sentido el uso del espacio territorial es relevante para poder entender que nuestro territorio, requiere una ordenación, por un lado, para mejorar la calidad de vida de las personas y por otro lado, para promover un desarrollo sustentable en estas comunidades rurales. El trabajo plantea el análisis del territorio, considerando el espacio geográfico que está siendo intervenido para la expansión agrícola, la ganadería y las actividades de servicio.

Para el levantamiento de información se contempla el mapeo, la realización de entrevistas a los lugareños de la zona, la elaboración de esquemas y cuadros para la presentación de la información y toma de fotografías de los paisajes del lugar. El manuscrito se encuentra estructurado de la siguiente forma; en el primer apartado se identifica y caracteriza la unidad de estudio, identificando las características del paisaje de la zona, en el segundo apartado se describen los usos del territorio, donde se encuentran las zonas de usos: agrícola, ganadero, natural, poblacional y caminos con pavimento, en tercer lugar se presenta una propuesta para la protección de los espacios naturales, se presentan algunas fotografías de la zona de estudio y se finaliza el trabajo con algunos alcances y conclusiones finales. Se adjunta, además, la bibliografía utilizada. 


\section{Estado de la cuestión}

El uso del suelo y el recurso escaso del agua se han trasformado en un tema de debate en la actualidad debido al escaso ordenamiento territorial que existe sobre todo en zonas rurales; por esta razón, existe la necesidad de buscar nuevos enfoques de trabajo que puedan contribuir a mejorar las problemáticas ambientales presentes en los territorios rurales. A manera de ejemplo, en el artículo diagnóstico Evaluación y Seguimiento: Sistema estructurado, Usos del suelo rural (Municipio de Medellin, 2004) se valora la importancia del suelo en las zonas rurales y como la economía del país colombiano depende del desarrollo de la agricultura y las actividades pecuarias en estas zonas rurales.

Esta realidad rural de Colombia se puede extrapolar al desarrollo rural de poblados rurales como es el caso de la localidad de Pedregal, comuna de Monte Patria, Región de Coquimbo, Chile, que es un lugar apartado de la ciudad donde se desconoce el uso del suelo y como las actividades humanas que se han distribuido en el espacio territorial.

Para Plaza (2016: 344) "los paisajes rurales o los paisajes agrarios han constituido una referencia central de la Geografía rural”. En este sentido, es importante mencionar cómo el estudio de caso presentado en la zona de Pedregal, contribuye a conocer a partir de los usos del suelo y la escasez del agua, como se han configurado estos espacios rurales, siendo notorias las trasformaciones que han experimentado, producto de las actividades agrarias que se han desarrollado en el espacio geográfico.

En este sentido, los actores sociales como el campesinado, las familias dueñas de las propiedades donde se ha instalado la industria agrícola y los crianceros han ido ocupando este espacio, otorgando un uso dependiendo de las actividades productivas que se desarrollan en cada área. Así, en los terrenos planos se ha instalado la actividad agrícola, a través de las plantaciones de parronales donde los campesinos trabajan para poder subsistir; en la parte alta de los cerros, los crianceros han debido lidiar con la escasez de pasto producto de lo escabroso de los cerros y la poca disponibilidad de forraje que crece en estas zonas.

La localidad de Pedregal es un espacio que está siendo constantemente transformado por actividades económicas que llegan al lugar desde afuera como también nuevas formas de desarrollo que vienen desde las familias dueñas de la tierra. Márquez (I999:II3) "menciona que se debe necesariamente considerar como una de sus tareas el ordenamiento 
territorial, el uso racional y sustentable de los espacios geográficos que lo integran”. En este sentido es importante destacar la importancia del ordenamiento territorial a través de los usos del suelo, como una forma de reconocer los diferentes usos del espacio con el fin de poder tomar buenas decisiones que puedan equilibrar el desarrollo y el cuidado de los recursos naturales.

En este contexto se ha presentado este trabajo llevado a cabo en Pedregal; la idea es reconocer los usos del suelo y el recurso escaso del agua que presenta esta zona geográfica, para reflexionar sobre la importancia de un desarrollo sustentable en las zonas rurales.

\section{Caracterización de la unidad de estudio: Pedregal un pueblo rural a los pies de la cordillera de Los Andes}

La localidad de Pedregal se encuentra ubicada en la comuna de Monte Patria, provincia del Limarí, Región de Coquimbo, Chile. Un pueblo situado a $30^{\circ} 51^{\prime} 12.7 I^{\prime \prime}$ latitud Sur y $70^{\circ} 42>40.43$ ” longitud Oeste, en una zona geográfica precordillerana cercana a la cordillera de Los Andes (Figura $\mathrm{N}^{\circ} \mathrm{I}$ ) 

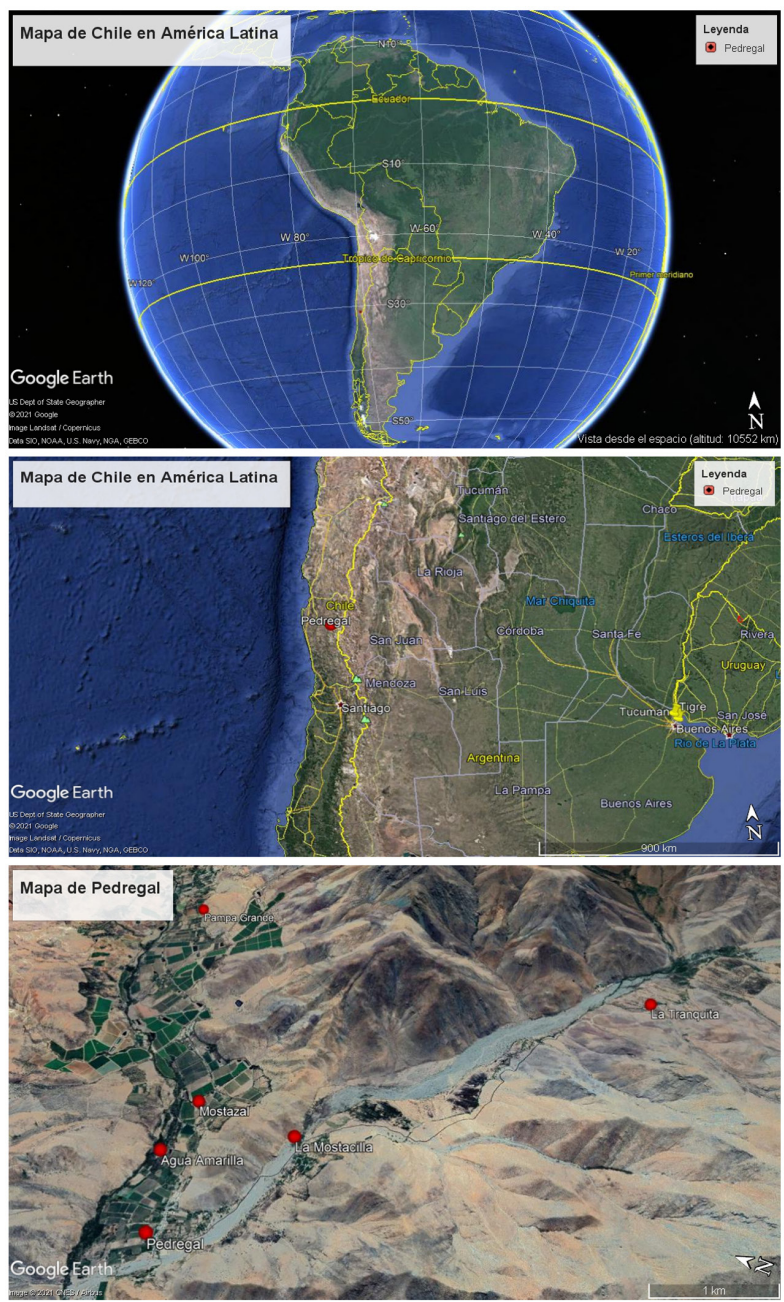

Figura 1. Localización del Pueblo de Pedregal, Chile.

Fuente: Elaboración propia en base a Google Earth (2020)

El pueblo cuenta con 364 habitantes entre los cuales 184 son mujeres y I80 son hombres (CENSO, 20I7). En el sector de Mostazal se concentra la mayor cantidad de parronales que existe en el valle. Según Guajardo 
(2018) la comuna de Monte Patria y Vicuña abarca el 65\% de la producción de uva de mesa de la región de Coquimbo. Considerando este aspecto, la actividad agrícola es muy importante en la zona, ya que entrega trabajo a muchas personas quienes se sustentan gracias a la existencia de esta producción.

La ganadería es una actividad que ha conseguido mantenerse en el tiempo. "En la Región de Coquimbo alrededor de 6.500 pobladores se dedican a la cría de cabras (GORE, 20I4). En los alrededores del pueblo de Pedregal existen majadas de cabreros, dentro de estas se pueden mencionar las majadas de los señores Ramón Cortés y Gustavo Tapia, que se ubican en el sector de La Mostacilla y en el sector de Agua Amarilla, donde se encuentra la majada de los señores Olivares. Estos cabreros dan sustento a sus familias con la venta de los quesos, los animales, pastando sus cabras en los potreros y los faldeos de las montañas del valle de mostazal. En la zona también existen personas dedicadas a la actividad de la minería en regiones más al norte de Chile.

\section{Actores locales que usan el espacio territorial de la localidad de Pedregal}

Pueden reconocerse tres tipos de actores locales que usan el espacio territorial de la localidad de Pedregal, que será analizado a continuación. Entre estos actores pueden mencionarse:

1. El campesinado. Lo componen principalmente personas dedicadas a la agricultura, desempeñando labores agrícolas en los fundos, como la corta de uva, la cosecha de paltas y duraznos.

2. Las familias dueñas de la tierra. Está formado por las familias dueñas de las propiedades que se dedican principalmente al cultivo agrícolas de variedades de uva, paltas y duraznos, este grupo permite dar trabajo a los campesinos quienes se encargan de producir.

3. Los crianceros o cabreros. Compuestos por personas pobres dedicados a la crianza del ganado caprino, estas personas, pastan sus cabras en la parte alta de los cerros y construyen sus majadas para poder manejar sus ganados. 


\section{Uso del espacio territorial en los alrededores de la localidad de Pedregal}

En los campos que rodean a la localidad de Pedregal los terrenos presentan diferentes usos como lo muestra la Figura 2, mapa sobre los usos del territorio en los terrenos que rodean a la localidad de Pedregal, comuna de Monte Patria, provincia del Limarí, Región de Coquimbo, Chile.

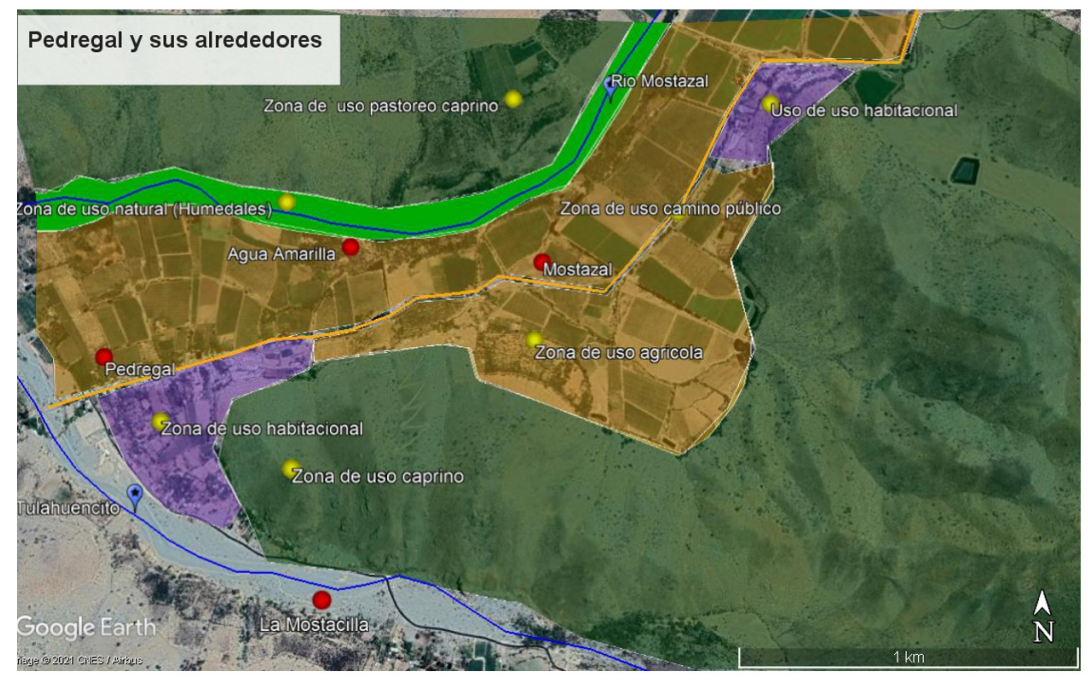

Figura 2. Usos del territorio en los terrenos que rodean a la Fuente: Elaboración localidad de Pedregal, comuna de Monte Patria, provincia propia (2020)

del Limarí, Región de Coquimbo. Chile

La zona de uso caprino es una zona a la que llegan las cabras de regreso de las verandas que se dan en la alta cordillera. Los animales, recorren los cerros que rodean a la localidad de Pedregal en busca de pastizales y brotes de arbustos verdes, como: colliguay, espinos, olivillos y algarrobos. "Los cabreros suben sus cabras a la cordillera los meses de octubre y noviembre, arreando su rebaño con sus familias. Los niños se quedan en la escuela y solo van personas mayores, debido a lo inhóspito del clima cordillerano" (Muñoz y Villaseñor Castro, 20I8:244).

Considerando estos antecedentes, las cabras solo están unos meses en este lugar, allí los crianceros con la ayuda de ramas de romero cons- 
truyen sus majadas y con ramas de espino los corrales donde dejan sus cabras cuando llegan del cerro.

La zona de uso natural (Humedales) corresponde a los ríos que pasan por los alrededores del pueblo de Pedregal, entre los que se encuentra el Río de Mostazal. En el valle podemos encontrar rasgos de dos tipos de humedales, el primero es un humedal ribereño que da origen a un bosque esclerófilo, y el segundo, un humedal alto andino que da origen a las vegas. Ambos resultan de vital importancia para el desarrollo humano y la mantención de los ciclos naturales, especialmente el ciclo del agua (Jorquera et al., 20I2). En este sentido, el Río del Valle de Mostazal es de vital importancia para el desarrollo de las actividades productivas que se dan en la zona mientras que los humedales que existen en el estero Tulahuencito, presentan una menor disponibilidad de agua que la del Río Mostazal; estos espacios naturales permiten la existencia de una biodiversidad que depende de la presencia de estos sitios.

La zona de uso agrícola, en la que están presentes las familias dueñas de la tierra, es una de las más importantes que existe en el lugar. En opinión de los lugareños, la actividad agrícola da trabajo a las personas y permite la subsistencia de los campesinos que viven de la producción y cosecha de la uva. La plantación de uva de mesa en las laderas de los cerros y en el fondo de los valles ha traído beneficios como la capacidad de contratar más mano de obra y, por otro lado, ha originado una problemática ambiental como lo es la escasez de agua para regar esas plantaciones, debido que hoy en día en la zona de Pedregal los años son cada vez menos lluviosos.

Analizando esta situación, es que el uso de estos terrenos está siendo modificado por la introducción de nuevas especies de uvas y otros cultivos que sean competitivos en el mercado. Sin embrago, hoy gran parte de la población es mano de obra de estos fundos agrícolas.

La zona de uso poblacional, donde viven campesinos y algunas familias dueńas de la tierra, se caracteriza por la existencia de distintos asentamientos humanos como Pedregal, Mostazal, por el sector de valle de Mostazal y por estero de Tulahuencito, el sector de La Mostacilla. El mayor número de personas se encuentra presente en el poblado de Pedregal; en esta localidad rural existe una iglesia, una escuela, un cuartel policial, un centro de salud de urgencia. En segundo lugar, se encuentra el sector de Mostazal en este sector existe una población y casas aisladas, allí la mayoría de la población de dedica a la labor agrícola en el fundo que está cercano al centro poblacional. 
En el estero de Tulahuencito existe una población muy escasa en el sector de La Mostacilla. Según el último CENSO (2017), registra 4I habitantes de las cuales 16 son hombres y 25 son mujeres. En esta zona, la actividad caprina es la principal actividad que da sustento a las familias que residen en el sector; la misma presenta un paisaje árido debido la ausencia de un río con un cauce permanente que favorece la existencia de un ambiente seco, donde el matorral arbustivo abierto es el alimento indispensable de las cabras.

La zona para uso de camino público es muy importante para la conectividad del pueblo con los otros sectores. Esta zona permite el libre tránsito de campesinos, comerciantes, dueńos de las tierra y crianceros, que caminan desde el sector de Mostazal hacia el pueblo de Pedregal. Este espacio geográfico contribuye a generar un polo de desarrollo para estas comunidades rurales, permitiendo dinamizar un comercio entre las agrícolas y las empresas que comercializan los productos como la uva de mesa, los cítricos y las paltas.

Recientemente, el turismo local ha cobrado fuerza en estas localidades apartadas de la ciudad, lo que ha hecho valorar las vías de comunicación terrestres que existen en estos lugares. De esta manera, el uso de los caminos públicos cobra importancia debido al rol que cumple en incentivar el desarrollo rural en estos lugares apartados. Este planteamiento puede ser apoyado con el testimonio de la presidenta de la Junta de Vecinos de la localidad de Los Morales de la Comuna de Monte Patria, cerca al valle de Mostazal, quien relata la importancia que tiene una obra de pavimentación para la comunidad: "Cuando llovía no se podía transitar por el barro y en el verano el problema era el polvo sobre todo porque en ese tiempo tenemos los comedores diarios afuera y no se podía comer por la tierra. Ahora para nosotros es un argullo y les damos las gracias a las personas que nos ayudaron" (Diario El Día, 2017). Los poblados rurales, como es el caso de Pedregal, se encuentran emplazados en lugares que presentan caminos de tierra; cuando llueve, estos se convierten en charcos o barriales, con la pavimentación. Esta problemática ha ido desapareciendo y ha mejorado la calidad de vida de las personas que viven en estos sectores aislados.

Un afecto negativo que se ha producido en estos espacios rurales intervenidos por el hombre con el fin de pavimentar los caminos ha sido la poca conciencia que han tenidos los conductores de los vehículos cuando se encuentran con flora y fauna autóctona. Los vehículos pasan más rápido 
y a veces las personas no respetan a los animales silvestres que utilizan estos pasos naturales para trasladarse de un lugar a otro; un ejemplo de esta situación es la experimentada por un Tucúquere que fue encontrado herido al costado de la carretera que une Illapel con Los Vilos.

$\mathrm{El}$ ave "presentaba problema en un ojo por lo que tuvo que ser sometida a diversos tratamientos para su recuperación". Según el director regional del Servicio Agrícola y Ganadero de la Región de Coquimbo en El Diario el Ovallino (2019), por las condiciones en que fue encontrado el animal "no tenemos certeza de lo que le puede haber pasado al ave, para quedar con su ojo herido y sin poder volar, pero dadas las lesiones y que encontraba a un costado de la carretera sospechamos que fue atropellada”.

Considerando esta problemática existente con el paso natural de la fauna silvestre de un lugar al otro lado de la carretera, es importante concientizar a las personas que al transitan por estas rutas deben hacerlo con el mayor cuidado posible y coordinar un trabajo en conjunto con la autoridad vial para trabajar con un plan que contemple la instalación de señaléticas y multas para quienes dañen los animales que utilizan estos senderos naturales.

\section{El registro de los diferentes usos del espacio territorial por zona}

La investigación realizada incorporó varias salidas a terreno con el fin poder dejar registro de los diferentes usos; se tomaron fotografías de las distintas zonas y se escribieron fichas para poder anotar los elementos que existían en la unidad de estudio.

\section{Zona de uso natural de humedales intervenida por actividades antrópicas}

En la primera fotografía se observa el camino que cruza la zona de bosque nativo de uso natural existente en el sector de agua amarilla, sector de Pedregal. Es importante notar como esta zona es atravesada por el camino que separa la zona cubierta por vegetación de los caminos construidos por el hombre (figura 3). En este sentido, esta problemática se puede extrapolar al humedal del Rio Lluta, en Arica, si bien el lugar es un Santuario de la Naturaleza, ha sido siempre un sitio vulnerable a acciones humanas. 


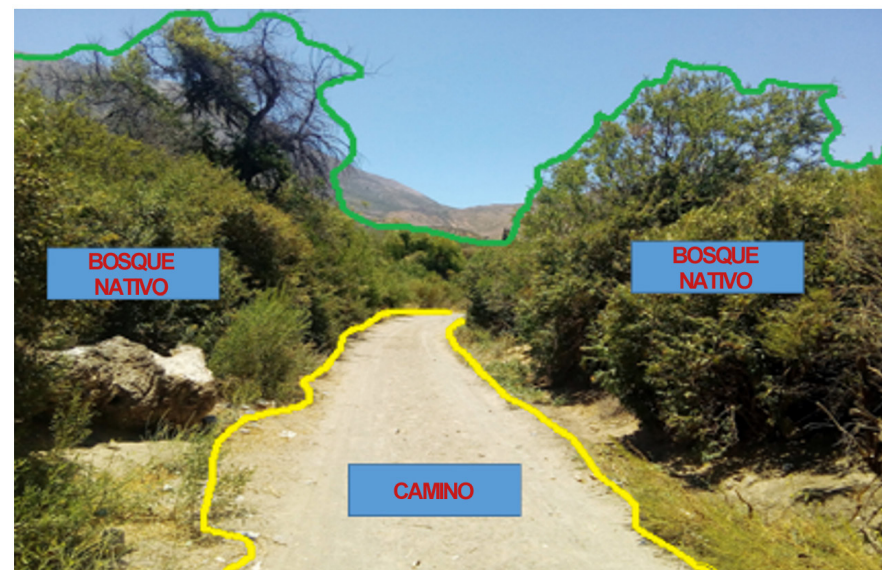

Figura 3. Zona de uso natural de humedales intervenida por Fuente: Elaboración actividades antrópicas, sector de Agua Amarilla, comuna de propia (2018) Monte Patria, provincia del Limarí, Región de Coquimbo, Chile.

"Ya en octubre 2006, por ejemplo, un vehículo de transporte militar dejo huellas en los totorales. Asimismo, tanto el municipio como la armada y carabineros han denunciado la existencia de fogatas, jaurías de perros salvajes, ingresos de vehículos particulares e instalación de campamentos de familias en el sector" (Astudillo, 20I8). Considerando estos aspectos en la zona de uso natural (Humedales) del Valle de Mostazal, las actividades antrópicas están degradando este espacio natural debido a la falta de una política clara de ordenamiento territorial que pueda ayudar a cuidar y proteger estas zonas naturales.

\section{Zona de uso para el pastoreo caprino y las actividades agrícolas}

Una segunda zona para analizar es la del pastoreo caprino. En la segunda fotografía se observa la limitación de las actividades agrícolas y los terrenos que son utilizados para el pastoreo de las cabras (figura 4). 


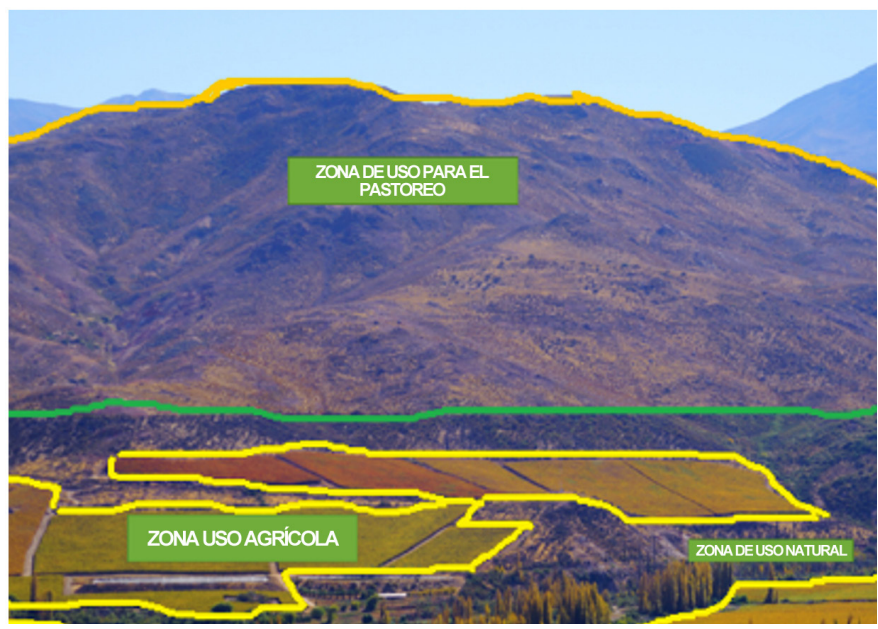

Fuente: Elaboración propia (2018).
Figura 4. Zona de uso para el pastoreo caprino y las actividades agrícolas, comuna de Monte Patria, provincia del Limarí, Región de Coquimbo, Chile.

La zona de uso para el pastoreo caprino se destaca porque las cabras "a medida que se introducen al monte realizan en forma alternada períodos de ingesta y períodos de descanso en tanto exploran el ambiente donde se encuentra pastoreando, hasta completar el recorrido que rutinariamente realizan durante la actividad de ingestión de alimentos" (Helguero, 2005).

La cabra es un animal que está acostumbrado a pastar en terrenos abiertos, especialmente en la Región de Coquimbo, esta actividad se ha extendido por décadas y se caracteriza por la existencia de trashumancia donde los cabreros llevan sus cabras a la cordillera en las estaciones de primavera-verano y otoño-invierno llegan con sus animales a las zonas precordilleranas y la costa. Sin embargo, las llegadas de empresas agrícolas a la zona de Pedregal han hecho aumentar la propiedad de la tierra de las familias que han adquirido las propiedades.

En este sentido, las empresas agropecuarias han ido restando espacio a las zonas de pastoreo caprino, provocando una disminución de las áreas de pastoreo, lo cual ha ido generando preocupación de parte de los cabreros, quienes cada año luchan por mantener sus ganados a pesar 
de que las áreas con mejores pastizales han comenzado a ser compradas para el cultivo agrícola.

\section{Zona de uso agrícola, zona de uso natural y la zona poblacional}

En la tercera fotografía se puede observar la limitación de la zona de sus agrícolas, el bosque esclerófilo de los humedales y la zona poblacional (figura 5).

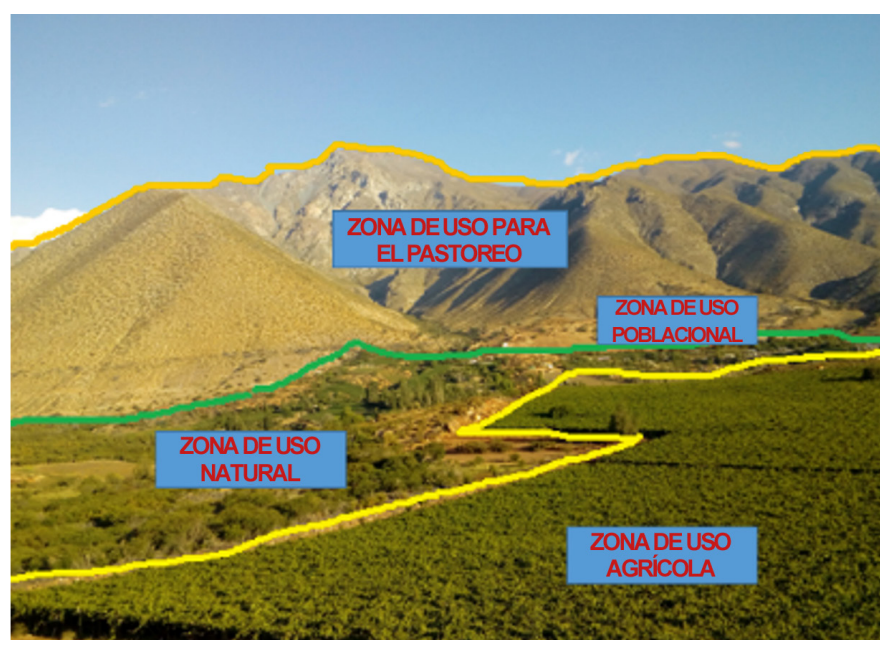

Figura 5. Zona de uso agrícola, zona de uso natural y la Fuente: Elaboración zona poblacional, Comuna de Monte Patria, provincia del propia (2018)

Limarí, Región de Coquimbo, Chile.

En relación con la limitación de las zonas agrícolas de la zona natural donde están presentes los humedales, existe cada día mayor interés de poder reducir estos espacios naturales sin considerar que el agua es un bien escaso y que requiere un mayor cuidado. Esta problemática generada a partir de la escasez del agua no ha sido tomada en cuenta por las familias dueñas de la tierra quienes han visto como una oportunidad de negocio la plantación de monocultivos sin considerar que el agua, cada año, sigue agotándose en el valle de Mostazal. Según Heselaars (2018), en la nota periodística Crisis del agua en Petorca: "los requerimientos hídricos no se condicen con la disponibilidad", el área de la 
quinta región es una las tres provincias declaradas como zonas de escasez vigente en el país.

El problema se acrecienta debido a la gran demanda hídrica que exigen los cultivos existentes en el lugar. Las zonas agrícolas en estos sectores periféricos rurales como es el caso de valle de Mostazal, comuna de Monte Patria, Región de Coquimbo, Chile, han experimentado un avance debido a la presencia de estos reservorios de agua natural que han logrado subsistir debido a la poca demanda de agua. En la actualidad, esta situación ha cambiado debido a la llegada de nuevas empresas agrícolas en la zona que buscan estos territorios para poder instalar sus fundos, esto ha provocado que las áreas naturales donde están presentes los humedales reduzcan su superficie y esto origine un problema por la disponibilidad de agua.

En el caso de las áreas poblacionales en el sector de Mostazal, no han tenido un crecimiento significativo, pero sí ha existido una mayor preocupación de la autoridad por mejorar la calidad de vida de los pobladores de estos sectores rurales. Esto se ha visto reflejado con la llegada de la pavimentación de los caminos y agua potable, por otro lado, la población sigue migrando a la cuidad debido a mejores oportunidades de trabajo.

\section{Valoración de los espacios y limitación de las fronteras; aspectos necesarios para el desarrollo sustentable en los alrededores de la localidad de Pedregal}

Los usos del territorio en los alrededores de la localidad de Pedregal se encuentran divididos en cinco usos: zona de uso agrícola, zona de uso para el pastoreo caprino, zona de uso natural (Humedales), zona uso poblacional y zona de uso de camino público. Considerando este aspecto, el territorio presenta una interrelación entre los distintos espacios donde se sitúan los distintos usos. En este sentido, la zona de uso natural (Humedal) está muy ligado a la zona de uso agrícola ya que el agua que sacan para regar las plantas de parras, paltos y cítricos la obtienen del Río Mostazal; por esta razón, se requiere incentivar una planificación que pueda estimar la cantidad de agua que se dispone para poder plantar sin alterar el orden de los ecosistemas naturales y las comunidades humanas existentes en la zona. 
Cuando no existe un trabajo colaborativo entre comunidad, actores locales y empresas agrícolas que apunte a cuidado responsable del agua y a crecimiento sostenible, podemos observar situaciones como es el caso del Valle de Petorca, donde no hubo un trabajo responsable frente a los usos de los recursos naturales. "El 30.5 por ciento de la producción de la fruta se genera en la provincia de Petorca a costa de los ríos y dejar sin agua para su consumo a las comunidades del territorio" (Velásquez, 20I8). Analizando la situación vivida en esta provincia de la Región de Valparaíso es necesario en el Valle de Mostazal incentivar buenas prácticas para el manejo del agua y así poder tener un crecimiento equitativo en esta unidad territorial.

La plantación de parronales y el uso de espacios para la instalación de casas en zonas de paso de aguas en tiempos de lluvia en estas zonas rurales, es muy común debido al escaso ordenamiento territorial que existe en estos lugares, sobrecoge la historia de una niña de io años del sector del Gallo, en la comuna de Andacollo, quien según relata la nota de El Día salió de su hogar a pedir ayuda, porque las aguas estaban a punto de llevarse la vivienda, pero no se percató de la crecida de una quebrada siendo arrastrada por la fuerza del agua (Diario El Día, 20I7).

Observando la situación acontecida en Andacollo, comuna cercana a Monte Patria, es importante una mayor fiscalización de parte de las autoridades sobre la construcción de viviendas por parte de la población. En la localidad de Pedregal existe poca población, el lugar se encuentra emplazado entre medio de dos ríos. Por un lado, se encuentra el estero Tulahuencito ya que es un cauce que solo trae agua en los años lluviosos, la ocurrencia de aluviones pone en peligro las viviendas que se encuentran en zonas de riesgo como son los sectores de Pedregal oriente y la conexión del pueblo con los otros sectores donde en ańos pasados el río pasó a llevar algunas casas y cortó el camino que une con la localidad.

Los terrenos que colindan con el Río Mostazal se encuentran con plantaciones de parronales y duraznos. En crecidas del río, durante los temporales del ańo 1997, las plantaciones en la zona del cauce natural del río, fueron arrastradas por la crecida provocando pérdidas a los agricultores de la zona. Es importante mencionar en esta situación la existencia de ordenanzas para regular las plantaciones. En este sentido, el límite de las zonas de riesgo que presenta el río no ha sido claro; por esta razón, algunos propietarios de los terrenos empiezan a plantar y cerrar 
espacios que presentan usos naturales como es el caso de los humedales naturales que cubren los ríos.

La destrucción de la vegetación asociada al cauce del río, tiene impactos negativos en la sobrevivencia de la flora y vegetación propia de la zona. Según el Informe país estado el medio ambiente en Chile 1999-2015 (Universidad de Chile, 20I6:I80) "los mayores contribuidores históricos a la pérdida del bosque nativo han sido la habilitación de los terrenos agrícolas, sustitución de plantaciones forestales, pérdida de bosques hacia matorrales e incendios forestales que en su mayor proporción son productos intencionalmente".

La compra de terrenos para uso agrícola está quitando terrenos a las zonas de pastoreo; esta acción es muy recurrente en la actualidad, debido al bajo precio que tienen los terrenos principalmente de comunidades agrícolas. Las empresas agrícolas limpian estos terrenos sustituyendo la vegetación nativa por plantas introducidas que demandan mucha agua como es el caso de las parras, cítricos y paltos, esta situación ha ido generando un problema en la limitación de los espacios de cada zona, la actividad caprina se ha ido trasladando hacia zonas donde los pastos son menos y la capacidad para poder alimentar este ganado cada día es menor. En este escenario que viven los cabreros se hace necesario destinar zonas para el pastoreo caprino con un instrumento de planificación territorial que pueda permitir la existencia de estas áreas en el tiempo, con el fin que la actividad de los crianceros permanezca en estos lugares.

\section{Reflexiones finales}

Con frecuencia enfocamos nuestra mirada en los recursos que nutren nuestros sistemas económicos. Sin embrago, el agua, la energía, los recursos forestales y mineros, la flora y fauna, por nombrar algunos, son los elementos que no están desvinculados del entorno del espacio temporal que los alberga (Precht et al., 2016). En este sentido, es importante entender que en el territorio estudiado es importante incentivar un trabajo que permita la existencia de zonas de uso natural, zonas de uso para el desarrollo de la ganadería caprina, zonas de uso para el desarrollo poblacional, zonas de uso para el desarrollo agrícola y espacios para la pavimentación de caminos con el fin de lograr en la comunidad del sector la importancia conocer cada espacio para la seguridad y el 
bienestar de esta comunidad rural. Según Gómez (2008) el objetivo final de la ordenación del territorio es el desarrollo sostenible de las unidades territoriales, a que se aplica, entendido en términos de calidad de vida y plasmado en el sistema territorial.

Considerando estos planteamientos es necesario recurrir a los planes de ordenamiento territorial para poder manejar de mejor manera los usos del territorio con el fin de evitar problemas que puedan originarse a través de desastres naturales y catástrofes ambientales que puedan suceder por no considerar la relevancia que tienen los espacios naturales para el equilibrio de los ecosistemas. En la estrategia de desarrollo de la región de Coquimbo, se plantea la existencia de planes de desarrollo con la participación de los actores locales, con el fin de lograr un mayor protagonismo de los actores locales; en este sentido, se plantea un trabajo colaborativo entre comunidad, institución pública y privada para lograr mejorar la gestión del territorio en los espacios rurales como es el poblado de Pedregal. 


\section{Bibliografía}

Astudillo, X. (2018). Polémica por proyecto de ruta en Arica que atravesara un humedal, La Tercera. [En línea] recuperado de: https://www.latercera.com/ nacional/noticia/polemica-proyecto-ruta-arica-atravesara-humedal/227845/

CENSO (2017). Datos estadísticos de la población de Monte Patria, provincia del Limari, Región de Coquimbo, Instituto Nacional de Estadisticas de Chile.

Diario El Día (2017). Así han sido las lluvias que han afectado a la región en los últimos 6o años, Diario El Día. [En línea] Recuperado de: http://www. diarioeldia.cl/region/tiempo/asi-han-sido-lluvias-que-han-afectado-regionen-ultimos-6o-anos/

Diario El Día (2018). Inauguran pavimentación de camino rural en Monte Patria, Diario El Día. [En línea] Recuperado de: http://www.diarioeldia.cl/ region/monte-patria/inauguran-pavimentacion-camino-rural-en-montepatrial

Diario El Ovallino (2019). Tras días de tratamiento el SAG libera a Tucúquere encontrado herido, Diario El Ovallino. [En línea] Recuperado de: http:// www.elovallino.cl/cronicas/tras-diez-dias-tratamiento-sag-libera-tucuquereencontrado-herido/

Fernández, J. (2018). Crisis de la uva afecta a pequeños productores, Diario El Día. [En línea] Recuperado de: http://www.diarioeldia.cl/region/ hechos-20I8/crisis-uva-afecta-pequenos-productores/

Gobierno de Chile (2018). Politica nacional de desarrollo rural, mejor calidad de vida y más oportunidades 20I4-2024, Santiago de Chile: Oficina de Estudios y Políticas Agrarias.

Gómez. D. (2008). Ordenamiento Territorial, 2 Edición, Madrid, Barcelona y México: Edición Mundi-Prensa.

GORE (2014). Trashumantes de la cordillera de Los Andes, los últimos pastores del hemisferio sur, Coquimbo: Corporación para el Desarrollo y la Integración Paso Agua Negra, Gobierno Regional de Coquimbo.

(2018). Estrategia de regional de desarrollo, Región de Coquimbo, Coquimbo: Gobierno Regional de Coquimbo.

Guajardo, D. (2018). Agricultores de uva estiman en US\$50 millones las pérdidas del sector y enfatizan que urge apoyo estatal, Diario El Día. [En línea] Recuperado de: http://www.diarioeldia.cl/economia/agricultura/agricultoresuva-estiman-en-usso-millones-perdidas-sector-enfatizan-que-urge/ 
Heselaars, T. (2018). Crisis del agua en Petorca. "Los requerimientos hídricos no se condicen con la disponibilidad", Emol Noticias. [En línea] Recuperado de: https://www.emol.com/noticias/Tecnologia/2018/03/29/900523/Crisisdel-agua-en-Petorca--Los-requerimientos-hidricos-no-se-condicen-con-ladisponibilidad.html

Helguero, P. (2005). Pastoreo caprino en el monte formoseño (Argentina), REDVET. Revista Electrónica de Veterinaria, Vol. VI, N II, pp. I-I4.

Jorquera, A.L.; Sánchez, F., Iturrieta, C.; Valdés, M.; Espinosa, M.; Espinosa, S. (2012). El valle en nuestras manos. La importancia de los humedales del rio Mostazal, Comuna de Monte Patria, Región de Coquimbo, Santiago de Chile: Tanque Editorial, 84 p.

Márquez Poblete, M.A. (I999). El ordenamiento territorial de los espacios rurales en Chile, Revista de Geografía Norte Grande, Vol. 26, pp. II3-II8.

Municipio de Medellín (2004). Diagnóstico, Evaluación y Seguimiento: Sistema estructurado, Usos del suelo rural. Alcaldía de Medellín, Medellín: Departamento Administrativo de Planeación. Compromiso de toda la ciudadanía. Documento técnico de soporte pot (Acuerdo 46, 2006).

Muńoz, E. J.; Villaseńor Castro, R. (2018). Usos de plantas nativas por una comunidad de cabreros en la quebrada de Tulahuén, Región de Coquimbo, Chile, Revista IDESIA, Vol. 36, N², pp. 243-258.

Parauelo, J.; Jobbágy, E.; Laterra, P.; Diaguez. H.; Garcia, A.; Paniza, A. (2014). Ordenamiento Territorial Rural: Conceptos, métodos y experiencias, Buenos Aires: Universidad de Buenos Aires, Ministerio de Agricultura, Ganadería y Pesca, Organización de Naciones Unidas para la Alimentación y Agricultura, $575 \mathrm{p}$.

Plaza, J. (2016). Valor patrimonial de los paisajes rurales, algunos ejemplos, Vera, J. F.; Olcina, J.; Hernández, M. (Eds.). Paisaje, cultura territorial y vivencia de la Geografia. Libro homenaje al profesor Alfredo Morales Gil, San Vicente del Raspeig: Publicaciones de la Universidad de Alicante, pp. 343-360.

Precht, A.; Reyes, S.; Salamanca, C. (2016). El ordenamiento territorial en Chile, Santiago de Chile: Ediciones Universidad Católica de Chile.

Universidad de Chile (2016). Informe pais, Estado del Medio Ambiente en Chile. Comparación I999- 2015, Santiago de Chile: Instituto de Asuntos Públicos, Centro de Análisis de Políticas Públicas.

Velásquez, F. (2018). Empresas de palta has reventaron ecosistemas y secaron Petorca, Diario Electrónico de la Universidad de Chile, Año XI. 